\title{
A Historical Note \\ On The Proof Of The Area Of A Circle
}

Yonah Wilamowsky, Seton Hall University, USA

Sheldon Epstein, Seton Hall University, USA

Bernard Dickman, Hofstra University, USA

\begin{abstract}
Proofs that the area of a circle is $\pi r^{2}$ can be found in mathematical literature dating as far back as the time of the Greeks. The early proofs, e.g. Archimedes, involved dividing the circle into wedges and then fitting the wedges together in a way to approximate a rectangle. Later more sophisticated proofs relied on arguments involving infinite sequences and calculus. Generally speaking, both of these approaches are difficult to explain to unsophisticated non-mathematics majors. This paper presents a less known but interesting and intuitive proof that was introduced in the twelfth century. It discusses challenges that were made to the proof and offers simple rebuttals to those challenges.
\end{abstract}

Keywords: Area; circle; history of mathematics; pedagogy

\section{INTRODUCTION}

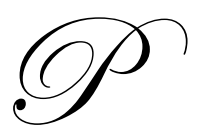

roofs that the area of a circle is $\pi \mathrm{r}^{2}$ can be found in mathematical literature dating as far back as the time of the Greeks. The early proofs, e.g. Archimedes, involved dividing the circle into wedges and then fitting the wedges together in a way to approximate a rectangle (see e.g. [1]). Later more sophisticated proofs relied on arguments involving infinite sequences and calculus. Generally speaking, both of these approaches are difficult to explain to unsophisticated non-mathematics majors. For this class of students, Epstein and Hochberg [2] outlined a less known but interesting and intuitive proof that was introduced in the twelfth century by Tosafot, a group of medieval rabbis who created critical and explanatory glosses on the Talmud (Garber and Tsaban [3] credit the proof to the $12^{\text {th }}$ Century mathematician, Rabbi Abraham bar Hiya in The Book of Mensuation of the Earth and its Division). Epstein and Hochberg do not, however, offer the subsequent history of this proof. The methodology was in fact challenged by counter-example in a late $17^{\text {th }}$ Century treatise. In this paper we review the original proof, the challenge and subsequent responses. We use simple spatial reasoning and elementary algebra to demonstrate that the "counter-example" supports the solution technique rather than contradicts it.

\section{THE PROOF}

Divide the circle of radius $r$ into $n-1$ concentric washers of equal width and a circle in the middle with radius $\mathrm{r} / \mathrm{n}$. Considering the circle in the middle as a washer with inner circle radius zero, we can view this as a system of $n$ washers. Diagram 1 is an example with $n=3$. Start by spreading out the outer washer (ring) so that it becomes flat. Its shape will be a trapezoid whose lower base equals the circumference of the outer washer, i.e. $2 \pi$ r, and whose upper base equals the circumference of the second outermost washer, i.e. $2 \pi(\mathrm{r}-\mathrm{r} / \mathrm{n})$. Similarly, spread the next washer so that it lies right above the previous. Its lower base will be the same length as the upper base of the previous one. Continue this process until all n washers have been "opened up".

The area of the original circle is equal to the sum of the areas of all the trapezoidal figures. As $n$ gets large, the height of each trapezoid gets small and the entire figure becomes a triangle with base $2 \pi r$ and with height $r$. Thus, says Tosafot, the area of the triangle is $(2 \pi r) r / 2=\pi r^{2}$. 


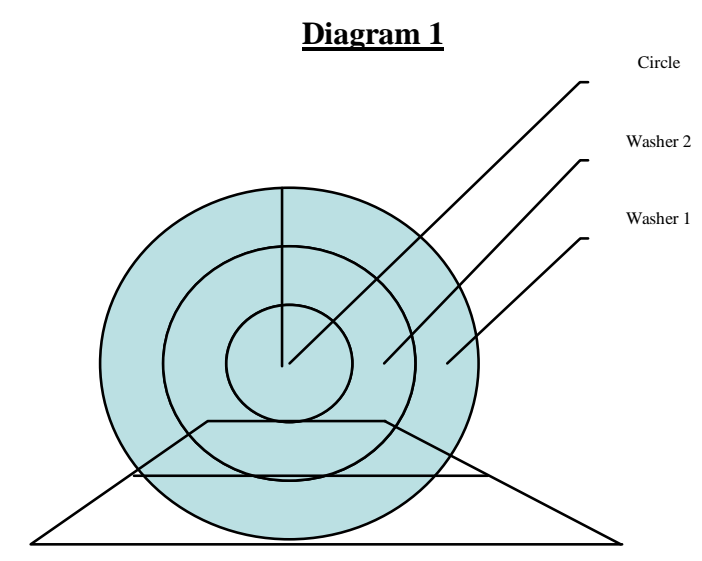

\section{THE COUNTER EXAMPLE}

Garber and Tsaban cite the following difficulty with this proof subsequently posed by Chavas Yair [4] in the $17^{\text {th }}$ century. Consider the same technique as applied to a square rather than a circle. The comparable picture (Diagram 2), using square rather than circular washers, would appear as follows:

\section{Diagram 2}

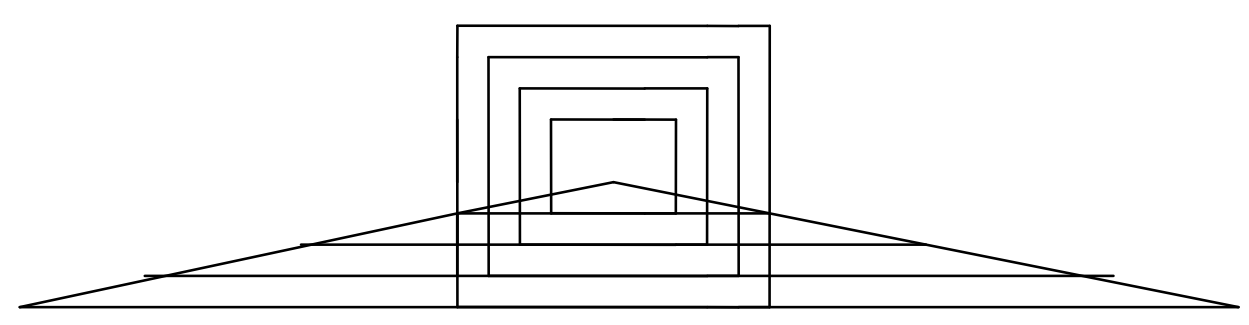

Using the same argument as before, the area of the square will be the same as the area of the triangle. If the side of the square is $s$, then the base of the triangle is $4 \mathrm{~s}$ and the height is $s / 2$. Thus the area of the triangle is $4 \mathrm{~s}(\mathrm{~s} / 2) / 2=\mathrm{s}^{2}$ as expected. However, suppose we look at the square oriented toward the diagonal (Diagram 3) and cut it toward the center along the diagonal as follows:

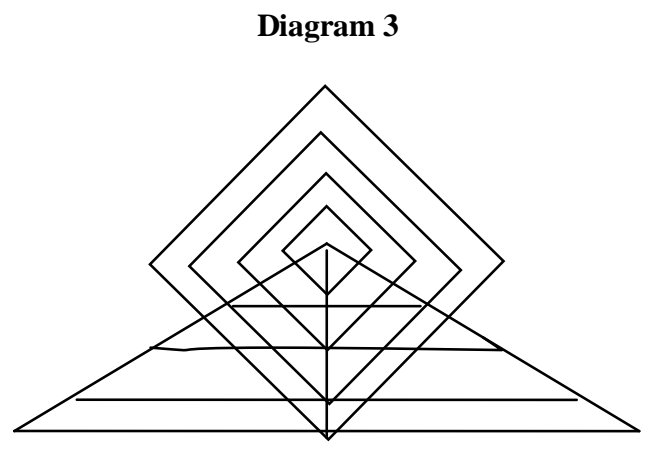

Spreading the concentric (square) washers along the diagonal now yields a triangle with a base of $4 \mathrm{~s}$ but with a height equal to the diagonal of the square, i.e. $\sqrt{2} \mathrm{~s} / 2$. Thus the area of the triangle is $\sqrt{2} \mathrm{~s}^{2}$. Chavas Yair offers 
a possible solution to the counter-example. He suggests that in the case of the square, as compared to the circle, opening the washers to straighten them out results in a number of wedges of empty space that reduce the overall area of the transformed figure. For example, looking at the left corner of the square, if we cut the corner on the bias in order to "open" it up:

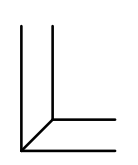

after spreading the vertical and horizontal parts we get:

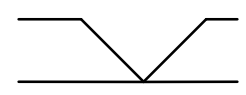

Spreading all four corners of the washer in a similar fashion we get

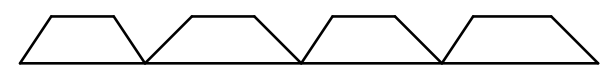

The wedges in this picture represent empty space at the corners when the washer is spread out. The resulting reorientation of the original square is therefore not a series of solid trapezoids but trapezoids with missing wedges. Each successive cut introduces 3 new missing wedges. Chavas Yair concludes that in the case of the square, it is possible that the introduction of these spaces into the resulting triangle accounts for the fact that the area of the triangle is greater than the original square. The circle does not have this problem.

However, Chavas Yair is not convinced by his own argument. He realizes that opening up the square in the original orientation, where the technique did give the correct area, also introduces the same wedges. In fact, he is concerned that if a circle is equivalent to a regular polygon with an infinite number of sides, then perhaps in the case of the circle, there are also minute wedges which are unaccounted for. On this basis, Chavas Yair questions whether the original technique of Tosafot is in fact correct, and perhaps the correct area was achieved fortuitously. While Chavas Yair himself recognizes the spatial impossibility of his counter-example, he remains unable to precisely identify the problem.

Bleicher [5] offers the following solution to the problem. He first recognizes that when "opening" the square washers, no matter which orientation is used, the height of the individual trapezoids must equal the width of the washer s/2n (i.e. in Diagram 4- $\boldsymbol{b}$ the space between the 2 horizontal lines), not its length along the diagonal $\sqrt{2}(\mathrm{~s} / 2 \mathrm{n})$ (i.e. in Diagram 4- $\boldsymbol{a}$ the length of the diagonal).

\section{Diagram 4}

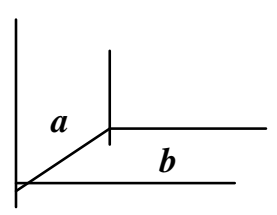

Therefore, the height of the resultant triangle will be one-half the side of the square and the area of this triangle is $n[s /(2 n)][4 s] / 2=s^{2}$. Bleicher notes that this resolves the difficulty introduced by the counter-example but does not address the wedge issue Chavas Yair subsequently introduced, i.e. if there are spaces included in the area of the triangle, why does the methodology yield the correct area? Bleicher then addresses these wedge spaces by considering what happens to them as the number of washers increases to infinity. He proves that as the number of inscribed square washers increase to infinity (i.e. $n \rightarrow \infty$ ) so do the number of wedges, but that at the same time the total area of these spaces is decreasing. He then demonstrates that the totality of the missing space is going to zero. 
Therefore, the area of the triangle is indeed equal to the area of the original square and there is no problem with the Tosafot technique.

\section{AN ALTERNATIVE SOLUTION}

A simple algebraic analysis of the case of the square shows that Bleicher's solution introduces unneeded complexity. Rather than posing a contradiction to the proof of Tosafot, the example of Chavas Yair can be used to help explain and validate the procedure.

It is easily seen that the outer washer of the square with side $\mathrm{s}$ which has been divided into $\mathrm{n}$ segments as before, can be opened to the figure of a rectangle. To demonstrate this rather than "opening" the washer by making diagonal cuts in the corners, make the cuts as indicated in Diagram 5.

\section{Diagram 5}

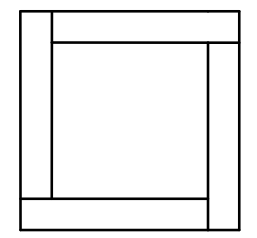

When the four pieces of the washer are placed adjacent to each other the result is a rectangle with length $4 s-4 s /(2 n)$ and width $s /(2 n)$. This is so since each corner of the washer is a square with side $s /(2 n)$. Similarly, the second outermost washer, when opened, is a rectangle with length $4 s-12 s /(2 n)$ and width $s /(2 n)$. When all $n$ washers are opened up in a similar fashion, the resulting picture is Diagram 6:

\section{Diagram 6}

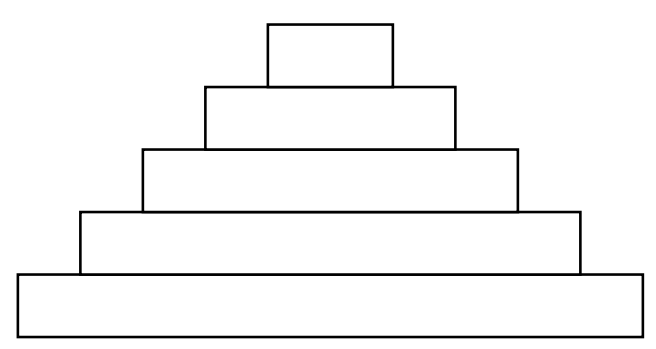

The sum of the areas of the $\mathrm{n}$ rectangles, for any value $\mathrm{n}$, is

$$
\begin{aligned}
& \mathrm{s} /(2 \mathrm{n})[(4 \mathrm{~s}-4 \mathrm{~s} / 2 \mathrm{n})+(4 \mathrm{~s}-12 \mathrm{~s} /(2 \mathrm{n})+\ldots+(4 \mathrm{~s}-4(2 \mathrm{n}-1) \mathrm{s} /(2 \mathrm{n})]= \\
& \mathrm{s} /(2 \mathrm{n})[4 \mathrm{sn}-4(1+3+5+\ldots+(2 \mathrm{n}-1)) \mathrm{s} /(2 \mathrm{n})]= \\
& \mathrm{s} /(2 \mathrm{n})\left[4 \mathrm{sn}-4 \mathrm{n}^{2} \mathrm{~s} /(2 \mathrm{n})\right]=\mathrm{s}^{2}
\end{aligned}
$$

It is therefore easily seen that the sum of the $\mathrm{n}$ rectangles is, as expected, $\mathrm{s}^{2}$, the area of the original square. As in the case of the circle, as the number of washers $n$ increases to infinity, the figure above will tend toward a triangle, with base $4 \mathrm{~s}$ and height $\mathrm{s} / 2$, whose area is $\mathrm{s}^{2}$. Far from the square being contradictory to the proof of the area of a circle, it can be used to support the argument in presentation to students. 


\section{AUTHOR INFORMATION}

Yonah Wilamowsky is a professor and former chairperson of computing and decision sciences at the Stillman School of Business, Seton Hall University. He holds a PhD in operations research from New York University. His research interests center on applications of statistics and quantitative methods to the law, business processes, biblical studies, and higher education. Among other journals, his work has appeared in Naval Research Logistics, Journal of the Operational Research Society, American Journal of Mathematical and Management Sciences, Tradition, Journal of College Teaching and Learning, Property Tax Journal, Location Sciences, and Computers and Operations Research.

Sheldon Epstein is a professor of computing and decision sciences at the Stillman School of Business, Seton Hall University. He holds a PhD in operations research from New York University and has done extensive business consulting. His research has appeared in: Computers and Operations Research, Naval Research Logistics, Journal of the Operational Research Society, American Journal of Mathematical and Management Sciences, Opsearch, Annals of the Society of Logistics Engineers, Journal of Property Tax Assessment and Administration, Property Tax Journal, Location Sciences, and Interface.

Bernard Dickman is an associate professor of information technology at the Zarb School of Business, Hofstra University. He holds a PhD in operations research from New York University and worked for many years as an operations research analyst for a major corporation. His research has appeared in Computers and Operations

Research, Naval Research Logistics, Journal of the Operational Research Society, American Journal of Mathematical and Management Sciences, OMEGA, The Mid-Atlantic Journal of Business, and Location Sciences.

\section{REFERENCES}

1. $\quad$ Bleicher, M., Maskil el David, Jerusalem, Israel, 1988. (In Hebrew).

2. Dunham, W. Archimedes' Determination of Circular Area. Ch. 4 in Journey through Genius: The Great Theorems of Mathematics, Wiley, New York, 1990, 84-112.

3. Epstein, S. and M. Hochberg, A Talmudic Approach to the Area of a Circle, Mathematics Magazine, September 1977, 210.

4. Garber, D. and B. Tsaban, A Mechanical Derivation Of The Area Of The Sphere, The American Mathematical Monthly, January 2001, 10-15.

5. Shalot Utshuvot Chavas Yair, Responsa 172, Jerusalem Edition, 1987 (in Hebrew). 


\section{NOTES}

